\title{
INFRARED OBSERVATIONS OF THE PLANETARY NEBULA NGC 7027
}

\author{
F.C. Gillett, F.J. Low, and W. A. Stein \\ (University of California, San Diego, University of Arizona, \\ and Rice University, U.S.A.)
}

An infrared spectrometer has been constructed with a resolution of $\Delta \lambda / \lambda \approx 0.02$ for observations in the wavelength range from 2.8 to $15 \mu$ using a gallium-doped germanium bolometer (Low, 1961) as the radiation-sensitive element. Observations of the planetary nebula NGC 7027 were made with this instrument in May and June 1967 at the Catalina Observing Station of the University of Arizona. It was discovered that there was a measurable continuum flux from this object in the wavelength range from 7.5-14 $\mu$ that compared in value with the strength of stellar radiation from $\alpha$ $\operatorname{Lyr}(\mathrm{A} 0)$ at about $\lambda=9 \mu$. These observations are described in detail elsewhere (Gillett et al., 1967).

The infrared observations of NGC 7027 show that the observed flux is almost two orders of magnitude larger than the expected infrared free-free continuum at $\lambda=10 \mu$. These results are perhaps similar to results of observations of the Orion nebula at $\lambda=5 \mu$ (Kleinmann and Low, 1967) that showed a flux 10 times larger than the expected free-free continuum from that nebula. NGC 7027 has also recently been observed at $\lambda=20 \mu$ (Low, private communication).

\section{References}

Gillett, F.C., Low, F.J., Stein, W.A. (1967) Astrophys. J., 149, L97.

Kleinman, D.D., Low, F.J. (1967) Astrophys. J., 149, L1.

Low, F.J. (1961) J. opt. Soc. Am., 51, 1300. 